\title{
The Planning of Electric Vehicle Charging Stations in the Urban Area
}

\author{
GE Shao-yun, FENG Liang, LIU Hong, WANG Long \\ Tianjin University, Nankai District, Tianjin, China \\ Email: xiaoxiao_1302@163.com
}

Keywords: electric vehicle; charging station planning; road network structure; distribution network

\begin{abstract}
With respect to the planning problems of electric vehicle charging stations in the urban area, the paper presents a planning model which considers the road network structure, vehicle flow information, distribution system structure and capacity constraints, etc. Under the relative constraints of distribution network, the model selects the station sites and plug-in locations with the objective of minimizing the sum of users' wastage cost on the way to the charging station and the investments of station lines, realizes the auto-partition of station service areas by the weighted Voronoi diagram, and also optimally allocates the station capacities based on the queuing theory. Finally, considering both the benefits of the power company and the electric vehicle users, the planning program optimization is finished by minimizing the costs of the whole society. The case studied shows that the presented methods and models are to some extent practical and reasonable to the optimal planning of electric vehicle charging stations in the urban area.
\end{abstract}

\section{Introduction}

With the increasing of environment pressures and resource shortages, energy saving is becoming more of a concern. Electric vehicles have become a focus of governments and enterprises, because they have the characteristics of energy saving and environmental protection. For the wide spread of electric vehicles, charging stations are the premise and foundation[1,2].

At present, many scholars have begun to study the charging behavior of electric vehicles and the planning and construction of charging stations. In [3], the impact of electric vehicles' charging on the power grid was analyzed in detail. In [4], the factors related to the demand on charging power of electric vehicles were analyzed. A statistical model of charging demand was built considering the probability distribution of some random factors. In [5], an optimized model for TOU power price time-period was proposed considering how to use ordered charging of electric vehicles to cut the peak and fill the valley of electricity power grid. In [6], Chinese current development of electric vehicle charging stations was described and the influencing factors and principles of charging station planning were proposed. In [7], the optimization cost model of locating and sizing of charging stations for electric vehicles was built. It simulated the number of electric vehicles based on the distribution of residents and conducted the sites planning at the target of maximizing charging stations' profits. In[8], the optimization model of charging mode choices was proposed, and the demand of each charging method could be forecasted based on this model. In [9], a two-step model was proposed: first converged the road information into 'demand clusters' by hierarchical clustering analysis and then applied optimization techniques to conduct the site planning, while certain constraints and cost were considered. In [10], a dynamic traffic network method was used to build a multi-objective optimization model with a hard time window constrains to obtain the optimal distribution and scale of charging stations. In [11], a model was proposed for the evaluation of charging station layout programs based on the game theory. Although these documents have built the mathematical model of electric vehicle charging station planning and defined a series of basic concepts, the combined effects of some important factors, such as the road network, the traffic flow, the structure and capacity constraints of distribution network, were less to be considered. Therefore, focusing on the impacts of these factors, an optimization model for urban charging stations planning was proposed in this paper. 


\section{The Mathematical Model}

\section{Planning Optimization Model}

It is necessary to consider the cost of construction and operation, but also consider the social benefits after the completion for the planning and construction of charging stations. Taking the interests of the power company and users into account, the social cost is used to select the optimal planning program. So the objective function can be expressed as the following formula:

$$
\min C=\sum_{i=1}^{N}\left(C_{1 i}+C_{2 i}+C_{3 i}+C_{4 i}+C_{5 i}\right)
$$

In the equation, $N$ is the number of charging stations. For the charging station $i, C_{1 i}$ is the construction investments; $C_{2 i}$ is the operation and maintenance cost; $C_{3 i}$ is the loss cost; $C_{4 i}$ is the charging cost of users in the service area; $C_{5 i}$ is the loss cost of users on the way to the charging station.

The construction investments of charging station $i$ :

$$
C_{1 i}=\left(e_{i} a+m_{i} b+c_{l} l_{i}+\omega_{i}\right) \frac{r_{0}\left(1+r_{0}\right)^{z}}{\left(1+r_{0}\right)^{z}-1}
$$

In the equation, $e_{i}$ is the number of transformers. $a$ is the price of the transformer. $m_{i}$ is the number of chargers. $b$ is the price of a charger. $l_{i}$ is the line length of the charging station $i$ access to distribution network. $c_{l}$ is the line price per kilometer. $\omega_{i}$ the cost of infrastructure. $r_{0}$ is the discount rate. $z$ is the operating period.

The operation and maintenance cost can be calculated in accordance with the percentage of the initial investment. Suppose $\eta$ is the percentage factor, the operation and maintenance cost of charging station $i$ :

$$
C_{2 i}=\left(e_{i} a+m_{i} b+c l_{i}+\omega_{i}\right) \cdot \eta
$$

The loss cost of charging station $i$ :

$$
C_{3 i}=e_{i}\left(C_{F e}+C_{C u}\right) \cdot T_{v} \cdot 365 \cdot p+m_{i}\left(C_{L}+C_{D}\right) \cdot k_{t} \cdot T_{v} \cdot 365 \cdot p
$$

In the equation, $C_{F e}$ is the iron loss and $C_{C u}$ is the copper loss of transformers. $C_{L}$ is the line loss in the charging station $i . C_{D}$ is the loss of a charger. $k_{t}$ is the ratio of chargers for working at the same time. $T_{v}$ is the effective charging time of the station. $p$ is the charging price

The charging cost of users in the service area:

$$
C_{4 i}=\sum_{j=1}^{N_{u m}} p \cdot q_{j} \cdot P_{v} \cdot 365
$$

In the equation, $N_{u m}$ is the number of charging demand nodes. $q_{j}$ is the number of electric vehicles at the charging demand node $j . P_{v}$ is the average capacity of electric vehicles.

There are two parts in the loss cost of users on the way to the charging station. One is the cost of power loss $h_{1}$ and another is the time cost $h_{2}$ :

$$
C_{5 i}=h_{1}+h_{2}=\frac{\sum L_{i}}{g} \cdot p \cdot 365+\frac{\sum L_{i}}{v} \cdot k \cdot 365=\sum_{j=1}^{N_{u m}} d_{i j} \cdot q_{j} \cdot\left(\frac{p}{g}+\frac{k}{v}\right) \cdot 365
$$

In the equation, $g$ is the mileage per kilowatt-hour. $k$ is the time cost of users in an hour [12] and $v$ is the average speed of electric vehicles. $d_{i j}$ is the distance between demand node $j$ and charging station $i$. $\sum L_{i}$ is the sum of the weighted distance from the charging station $i$ to the demand nodes in the service area.

\section{Sizing and Locating Model}

The location selection of the charging station is to consider two factors: the users' convenience and the line cost of the charging station access to the distribution network. The locating objective function can be expressed as the following formula: 


$$
\min C_{\text {stationi }}=c_{l} \cdot l_{i} \cdot \frac{r_{0}\left(1+r_{0}\right)^{z}}{\left(1+r_{0}\right)^{z}-1}+C_{5 i}
$$

The charging service of the charging station is random. Therefore, the optimization of chargers can be solved by using the queuing theory[13].

\section{Distribution Network Constraints}

Distribution network constraints include equality constraints and inequality constraints. Equality constraints refer to the flow equations. Inequality constraints are to consider some factors, such as the capacity limit and the safe operation of the distribution network.

a. Substation capacity constraints

$S_{i} \leq S_{i \max }$

In the Inequality, $S_{i}$ is the load of the substation. $S_{\text {imax }}$ is the maximum load that the substation can carry.

b. Charging power constraints

$$
\sum_{i=1}^{N} P_{C i} \leq P_{C}^{\max }
$$

In the Inequality, $P_{C i}$ is the charging power of the station $i . P_{C}^{\max }$ is the maximum charging power that the distribution network can accept.

c. Node voltage amplitude constraints

$V_{i}^{\min } \leq V_{i} \leq V_{i}^{\max }, i=1,2, \ldots, M$

In the Inequality, $V_{i}$ is the voltage amplitude of the node $i . V_{i}^{\max }$ and $V_{i}^{\min }$ are the maximum and minimum voltage amplitude of the node $i . M$ is the number of nodes in the distribution network.

d. Feeder maximum current constraints

$\left|I_{i j}\right| \leq I_{i j \max }, \quad i, j=1,2, \ldots, M$

In the Inequality, $I_{i j}$ is the current and $I_{i j m a x}$ is the maximum current of the feeder $i j$.

e. Access point capacity constraints

$P_{C i j} \leq P_{j \max }$

In the Inequality, $P_{c i j}$ is the charging power of the charging station $i$ which connects to the grid node $j . P_{\text {jmax }}$ is the maximum charging power that the node $j$ can accept.

\section{The Overall Planning Process}

The general idea of this paper is as follows:

First, the minimum and maximum number $\left(N_{\min }\right.$ and $\left.N_{\max }\right)$ of charging stations can be estimated in accordance with the maximum and minimum capacity $\left(S_{\max }\right.$ and $\left.S_{\min }\right)$ of the charging station [14]. So $N_{\text {min }} \leq N \leq N_{\text {max }}$.

Second, we can find an optimal planning program for each $N$. During the process, distribution network constraints should be considered. The initial locations are determined by using coordinate method [15]. The weighted Voronoi diagram is used to partition the service area [16]. The sites and access locations were chosen at the aim of minimizing the sum of the users' wastage cost on the way to the charging station and the investments of station lines. The allocations of charging stations were optimized by using the queuing theory.

Finally, taking the interests of the power company and users into account, we select the optimal planning program from the $N_{\max }-N_{\min }+1$ programs to minimize the social cost.

\section{Case Study}

In order to illustrate the methods and models, a study case is constructed. There are 48 intersection points, 110 road sections in the planning area which is $63 \mathrm{~km}^{2}$. The regional road network is shown in Fig. 1. The node coordinates and typical traffic data are shown in Table 1 . There are 3 substations 
(35/10kV, $2 \times 16 \mathrm{MVA})$ and 32 load nodes in the planning area. The network structure is shown in Fig.

2. The coordinates and load of the distribution nodes are shown in Table 2.

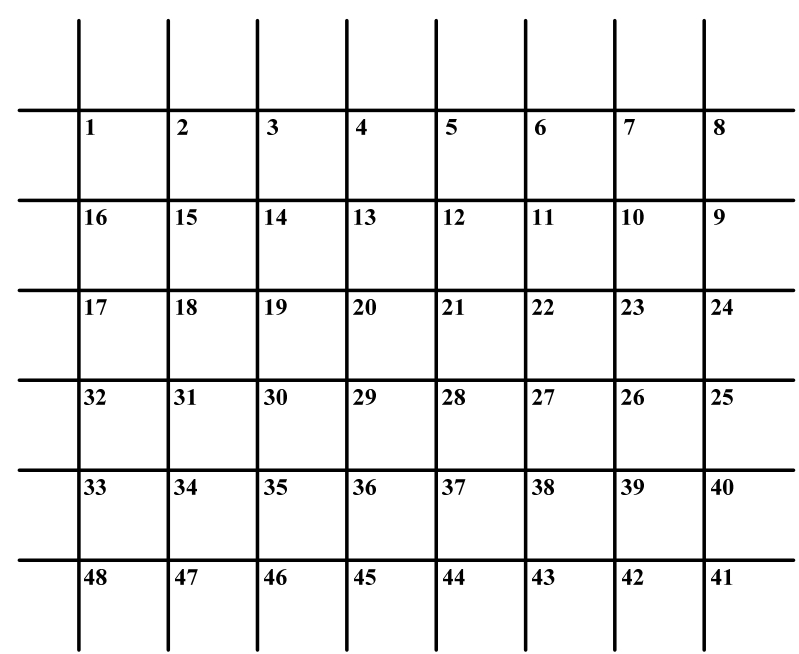

Fig. 1 The road network of the planning area

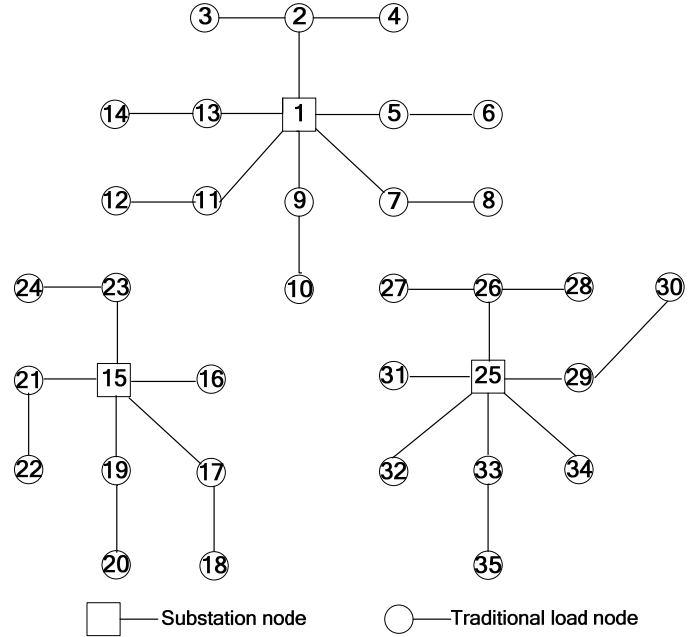

Fig. 2 The distribution network of the planning area

Table. 1 The coordinates and traffic flow of each intersection node

\begin{tabular}{|c|c|c|c|c|c|c|c|c|c|c|c|}
\hline No. & $\mathrm{X}$ & $\mathrm{Y}$ & Traffic Flow & No. & $\mathrm{X}$ & $\mathrm{Y}$ & Traffic Flow & No. & X & Y & Traffic Flow \\
\hline 1 & 1 & 6 & 8990 & 17 & 1 & 4 & 4068 & 33 & 1 & 2 & 6110 \\
\hline 2 & 2 & 6 & 5626 & 18 & 2 & 4 & 1918 & 34 & 2 & 2 & 4370 \\
\hline 3 & 3 & 6 & 7190 & 19 & 3 & 4 & 2484 & 35 & 3 & 2 & 2654 \\
\hline 4 & 4 & 6 & 6986 & 20 & 4 & 4 & 6080 & 36 & 4 & 2 & 5132 \\
\hline 5 & 5 & 6 & 10940 & 21 & 5 & 4 & 5320 & 37 & 5 & 2 & 7052 \\
\hline 6 & 6 & 6 & 8608 & 22 & 6 & 4 & 4198 & 38 & 6 & 2 & 8420 \\
\hline 7 & 7 & 6 & 5368 & 23 & 7 & 4 & 2628 & 39 & 7 & 2 & 6802 \\
\hline 8 & 8 & 6 & 8200 & 24 & 8 & 4 & 3424 & 40 & 8 & 2 & 8658 \\
\hline 9 & 8 & 5 & 8658 & 25 & 8 & 3 & 4024 & 41 & 8 & 1 & 8400 \\
\hline 10 & 7 & 5 & 4808 & 26 & 7 & 3 & 2870 & 42 & 7 & 1 & 5476 \\
\hline 11 & 6 & 5 & 8420 & 27 & 6 & 3 & 4312 & 43 & 6 & 1 & 9022 \\
\hline 12 & 5 & 5 & 6816 & 28 & 5 & 3 & 6422 & 44 & 5 & 1 & 6432 \\
\hline 13 & 4 & 5 & 4530 & 29 & 4 & 3 & 5086 & 45 & 4 & 1 & 2986 \\
\hline 14 & 3 & 5 & 2654 & 30 & 3 & 3 & 2484 & 46 & 3 & 1 & \\
\hline 15 & 2 & 5 & 4170 & 31 & 2 & 3 & 2118 & 47 & 2 & 1 & \\
\hline 16 & 1 & 5 & 6110 & 32 & 1 & 3 & 4264 & 48 & 1 & 1 & 562 \\
\hline
\end{tabular}

Table.2 The coordinates and load of each distribution network node

\begin{tabular}{|c|c|c|c|c|c|c|c|c|c|c|c|}
\hline No. & X & Y & Load $[\mathrm{MW}]$ & No & X & Y & Load [MW] & No. & X & Y & Load [MW] \\
\hline 1 & 4.5 & 5.5 & 0 & 13 & 3.5 & 5.5 & 0.70 & 25 & 6.5 & 2.5 & 0 \\
\hline 2 & 4.5 & 6.5 & 0.90 & 14 & 2.5 & 5.5 & 1.25 & 26 & 6.5 & 3.5 & 0.85 \\
\hline 3 & 3.5 & 6.5 & 1.15 & 15 & 2.5 & 2.5 & 0 & 27 & 5.5 & 3.5 & 0.95 \\
\hline 4 & 5.5 & 6.5 & 1.05 & 16 & 3.5 & 2.5 & 2.40 & 28 & 7.5 & 3.5 & 0.75 \\
\hline 5 & 5.5 & 6.5 & 1.10 & 17 & 3.5 & 1.5 & 1.15 & 29 & 7.5 & 2.5 & 1.35 \\
\hline 6 & 6.5 & 4.5 & 1.20 & 18 & 3.5 & 0.5 & 1.35 & 30 & 8.5 & 3.5 & 0.65 \\
\hline 7 & 5.5 & 4.5 & 0.75 & 19 & 2.5 & 1.5 & 1.60 & 31 & 5.5 & 2.5 & 2.10 \\
\hline 8 & 6.5 & 4.5 & 1.35 & 20 & 2.5 & 0.5 & 2.20 & 32 & 5.5 & 1.5 & 2.20 \\
\hline 9 & 4.5 & 4.5 & 0.85 & 21 & 1.5 & 2.5 & 1.50 & 33 & 6.5 & 1.5 & 1.05 \\
\hline 10 & 4.5 & 3.5 & 1.15 & 22 & 1.5 & 1.5 & 0.60 & 34 & 7.5 & 1.5 & 0.85 \\
\hline 11 & 3.5 & 4.5 & 0.65 & 23 & 2.5 & 3.5 & 1.50 & 35 & 6.5 & 0.5 & 0.55 \\
\hline 12 & 2.5 & 4.5 & 1.40 & 24 & 1.5 & 3.5 & 0.40 & & & & \\
\hline
\end{tabular}

At the time of target year, the percentage of the electric vehicle is $15 \%$. The capacity of each electric vehicle is $50 \mathrm{kWh}$. The power of every single charger is $96 \mathrm{~kW}$; there are at least 6 , at most 30 chargers in the station; the charger's efficiency is 0.9 , the coincidence factor is 0.9 ; the electric vehicle can run $7 \mathrm{~km}$ in each kilowatt-hour; the electricity price is $0.8 \mathrm{Yuan} / \mathrm{kWh}$; the average speed 
of electric vehicles is $20 \mathrm{~km} / \mathrm{h}$; and the time cost of users is $17 \mathrm{Yuan} / \mathrm{h}$.

The process of calculation is shown as below:

a. To estimate the number of charging stations: $N_{\min }=5, N_{\max }=20$.

b. To calculate the costs of the $N_{\max }-N_{\min }+1$ programs, the results are shown in Table 3 .

c. It can be seen from Table 3, when the number of charging stations is 7, that the social cost of the planning program is the smallest (72.91 million Yuan).

d. To determine the optimal sites, capacities, service areas and acess locations of the 7 charging stations. So the sites and capacities are shown in Table 4. The layout and service areas are shown in Fig. 3 and the access locations are shown in Fig. 4.

Table. 3 The social cost of each planning program [Unit: Ten thousand Yuan]

\begin{tabular}{|c|c|c|c|c|c|c|}
\hline$N$ & $C_{1}$ & $C_{2}$ & $C_{3}$ & $C_{4}$ & $C_{5}$ & $C$ \\
\hline 5 & 160.30 & 151.11 & 800.31 & 6004.91 & 206.39 & 7323.03 \\
\hline 6 & 171.86 & 162.01 & 801.20 & 6004.91 & 151.87 & 7291.85 \\
\hline 7 & 169.95 & 160.21 & 816.83 & 6004.91 & 138.67 & 7290.58 \\
\hline 8 & 192.17 & 181.16 & 825.75 & 6004.91 & 129.11 & 7333.10 \\
\hline 9 & 201.52 & 189.97 & 834.23 & 6004.91 & 118.84 & 7349.47 \\
\hline 10 & 204.06 & 192.36 & 835.12 & 6004.91 & 113.96 & 7350.41 \\
\hline 11 & 222.13 & 209.40 & 835.56 & 6004.91 & 106.62 & 7378.63 \\
\hline 12 & 229.60 & 216.44 & 850.30 & 6004.91 & 100.24 & 7401.51 \\
\hline 13 & 244.17 & 230.18 & 851.19 & 6004.91 & 95.17 & 7425.62 \\
\hline 14 & 240.62 & 226.83 & 859.23 & 6004.91 & 94.46 & 7426.04 \\
\hline 15 & 256.95 & 242.23 & 866.82 & 6004.91 & 88.83 & 7459.75 \\
\hline 16 & 261.14 & 246.18 & 874.41 & 6004.91 & 83.69 & 7470.34 \\
\hline 17 & 276.64 & 260.79 & 882.89 & 6004.91 & 76.98 & 7502.22 \\
\hline 18 & 291.69 & 274.97 & 876.63 & 6004.91 & 75.54 & 7523.74 \\
\hline 19 & 298.67 & 281.55 & 883.78 & 6004.91 & 73.65 & 7542.57 \\
\hline 20 & 303.14 & 285.77 & 898.52 & 6004.91 & 76.93 & 7569.26 \\
\hline
\end{tabular}

Table. 4 The locations and capacities of the charging stations

\begin{tabular}{|c|c|c|c|c|}
\hline No. & $\begin{array}{c}\text { X-coordinate } \\
\text { of the Station }\end{array}$ & $\begin{array}{c}\text { Y-coordinate } \\
\text { of the Station }\end{array}$ & The Number of chargers & The Road Nodes in the Service \\
\hline$S_{1}^{\prime}$ & 1.79 & 5.37 & 18 & $1,2,3,14,15,16,17,18,19$ \\
\hline$S_{2}^{\prime}$ & 4.16 & 1.53 & 14 & $35,36,37,44,45,46$ \\
\hline$S_{3}^{\prime}$ & 7.10 & 5.05 & 19 & $7,8,9,10,11,22,23,24$ \\
\hline$S_{4}^{\prime}$ & 1.30 & 1.63 & 14 & $31,32,33,34,47,48$ \\
\hline$S_{5}^{\prime}$ & 7.00 & 1.92 & 24 & $25,26,27,38,39,40,41,42,43$ \\
\hline$S_{6}^{\prime}$ & 5.00 & 6.00 & 15 & $4,5,6,12$ \\
\hline$S_{7}^{\prime}$ & 4.29 & 3.72 & 13 & $13,20,21,28,29,30$ \\
\hline
\end{tabular}

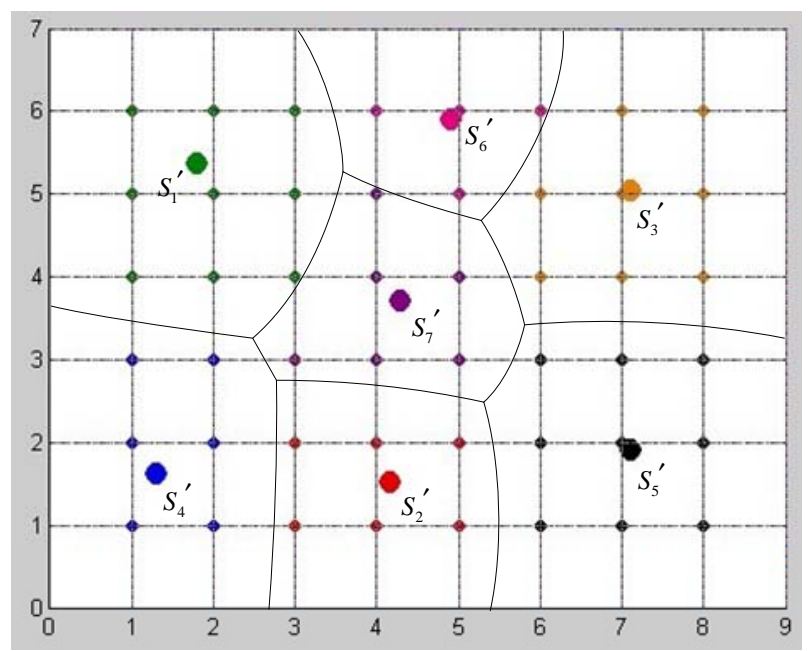

Fig. 3 The layout and the service areas of the charging stations 


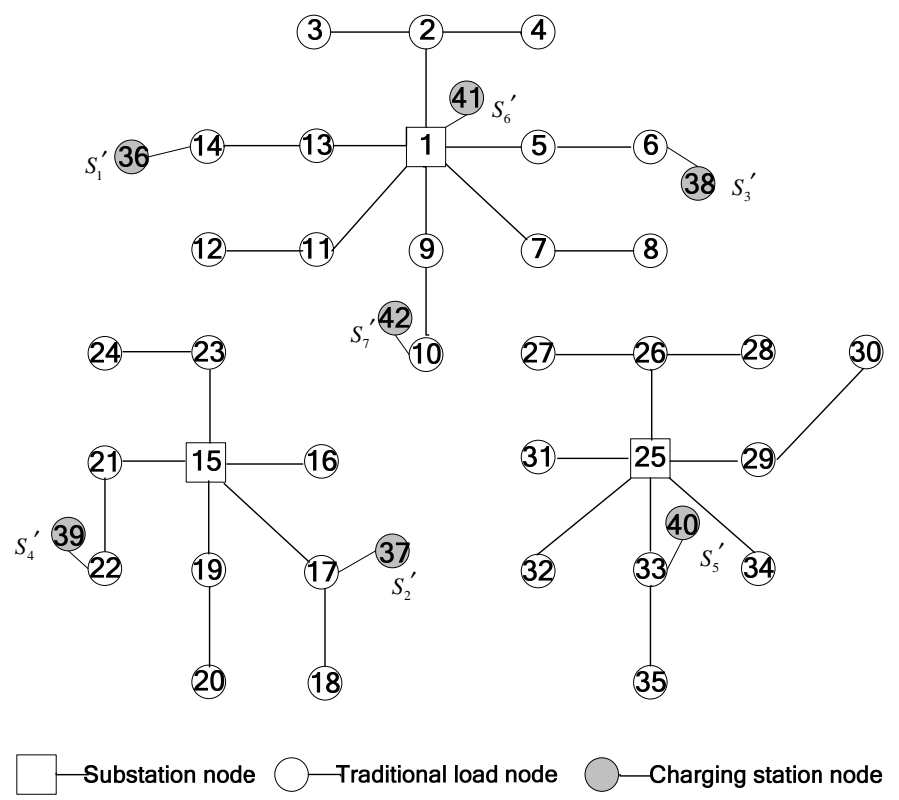

Fig. 4 The access locations of the charging stations

\section{Summary}

From the case study we can see that the road network and the traffic flow can directly affect the sites and service areas of the charging stations. And then the capacities will be indirectly affected by these factors. However, the distribution network structure and capacity constraints will have certain restrictions on the access locations, the number and capacities of the charging stations. Furthermore, they will have the influence on the sites and service areas and ultimately affect the social cost of the planning program. The impacts of these factors are interrelated. So the models and methods presented in this paper are mainly to coordinate the influence of these factors in order to find the optimal planning program. And the results of the example showed that the methods and models were feasible and reasonable for charging stations planning in the urban area.

\section{References}

[1] ZHANG Wen-liang, WU Bin, LI Wu-feng, et al. Discussion on Development Trend of Battery Electric Vehicles in China and Its Energy Supply Mode. Power System Technology, Vol.33, No.4, p. 1-5 (2009).

[2] SONG Yonghua, YANG Yuexi, HU Zechun. Present Status and Development Trend of Batteries for Electric Vehicles. Power System Technology, Vol.35, No.4, p. 1-7 (2011).

[3] GAO Ciwei, ZHANG Liang. A Survey of Influence of Electrics Vehicle Charging on Power Grid. Power System Technology, Vol.35, No.2, p. 127-131 (2011).

[4] Tian Liting, Shi Shuanglong, Jia Zhuo. A Statistical Model for Charging Power Demand of Electric Vehicles. Power System Technology, Vol.34, No.11, p. 126-130 (2010).

[5] GE Shao-yun, HUANG Liu, LIU Hong. Optimization of peak-valley TOU power price time-period in ordered charging mode of electric vehicle. Power System Protection and Control, Vol.40, No.10, p. 1-5 (2012).

[6] Xu Fan, Yu Guoqing, Gu Linfeng, and Zhang Hua. Tentative analysis of layout of electrical vehicle charging stations. East China Electric Power, Vol. 37, No. 10, p. 1672-1678, (2009).

[7] Kou Lingfeng, Liu Zifa, Zhou Huan. Modeling algorithm of charging station planning for regional electric vehicle. Modern Electric Power, Vol. 27, No. 4, p. 44-48, (2010).

[8] Wu Chunyang, Li Canbing, Du Li, and CAO Yijia. A method for electric Charging infrastructure planning. Automation of Electric Power System, Vol. 34, No.24, p. 36-39, (2010). 
[9] Andy Ip, Simon Fong, Elaine Liu. Optimization for Allocating BEV Recharging Stations in Urban Areas by Using Hierarchical Clustering. The 2nd International Conference on Data Mining and Intelligent Information Technology Applications (ICMIA 2010), Seoul, Korea, 2010.

[10] REN Yulong, SHI Lefeng, ZHANG Qian, HAN Weijian and HUANG Shoujun. Optimal Distribution and Scale of Charging Stations for Electric Vehicle. Automation of Electric Power System, Vol. 35, No. 14, p. 53-57, (2011).

[11] ZHOU Hongchao, LI Haifeng. Optimization Model of Electric Vehicle Charging Station Siting Based on Game Theory. Science Technolog y and Indust ry, Vol. 11, No. 2, p. 51-54, (2011). [12] QI Tongyan, LIU Dongmei, LIU Ying. A Study of the Traveling Cost of Beijing Residents. Journal of Highway and Transportation Research and Development, Vol. 25, No. 6, p. 144-146, (2008).

[13] LI Ruqi, SU Haoyi. Optimal Allocation of Charging Facilities for Electric Vehicles Based on Queuing Theory. Automation of Electric Power System, Vol. 35, No. 14, p. 58-61, (2011).

[14] Ge Shaoyun, Feng Liang, Liu Hong. The planning of electric vehicle charging station based on Grid partition method. 2011 International Conference on Electrical and Control Engineering (ICECE2011), p. 2726-2730 (2011).

[15] Ge Shaoyun, Li Hui, Liu Hong. Substation optimization planning based on the weighted Voronoi diagram. Automation of Electric Power Systems, Vol. 31, No. 3, p. 29-34, (2007).

[16] LU Zhi-ying, GE Shao-yun, WANG Cheng-shan. Substation Location Planning of the Weighted Voronoi Diagram Based on Particle Swarm Optimization Algorithm. Proceedings of the CSEE, Vol. 29, No. 16, p. 35-41, (2009). 